\title{
A prospective study of acceptability, safety and demographic profile for post placental intrauterine contraceptive device
}

\author{
Neha Jain*, Hemangi K. Choudhari
}

Department of Obstetrics and Gynecology, Seth G.S. Medical College and KEM Hospital, Maharashtra, India

Received: 08 May 2020

Accepted: 01 June 2020

\section{*Correspondence:}

Dr. Neha Jain,

E-mail: neha.jain1008@gmail.com

Copyright: (c) the author(s), publisher and licensee Medip Academy. This is an open-access article distributed under the terms of the Creative Commons Attribution Non-Commercial License, which permits unrestricted non-commercial use, distribution, and reproduction in any medium, provided the original work is properly cited.

\section{ABSTRACT}

Background: The objective of this study was to study the acceptance rate, safety, demographic profile and complication of post placental intrauterine contraceptive device (PPIUCD).

Methods: Study was prospective, interventional study, held in department of Obstetrics and Gynaecology at authors tertiary care centre. Number of participants was 280 . Counselling about family planning was done in antenatal OPD and indoor ward. Total duration of study was 18 months. Selection of participants done based on selection criteria. Participants who accepted postplacental intrauterine contraceptive device as method of contraception followed up to 6 weeks postnatal for complication related to PPIUCD.

Results: Authors noted that acceptance rate of PPIUCD was $13.6 \%$ and main reason for acceptance was its long acting reversible nature and main reason for denial was 'do not want contraception immediately'. In present study there was statistically significant association between parity and birth order. However, literacy, urban locality and employment had positive influence over acceptance. If good technique of insertion will be used than expulsion rate will also reduce as seen in present study i.e. expulsion rate only $2.6 \%$.

Conclusions: Post placental intrauterine contraceptive device is one of the best long acting reversible contraceptive methods. It does not affect breast milk production. Woman does not need extra visit to clinic for contraception and she is ensured that she has adequate contraception before getting discharge from hospital. PPIUCD provide adequate birth spacing between two children which reduces maternal and child morbidity.

Keywords: Acceptance rate, safety, Demographic profile, Post placental intrauterine contraceptive device

\section{INTRODUCTION}

Post-placental intrauterine contraceptive device (PPIUCD) insertion refers to the insertion of IUCD within 10 minutes of expulsion of placenta following a vaginal delivery or after caesarean section before closure of uterine incision. Intrauterine contraceptive device is one of effective long acting reversible, cotius independent contraceptive method.

IUDs provide a high level of efficacy in the absence of systemic metabolic effects to women, and no on-going motivation is required to ensure efficacy once the device has been placed. ${ }^{1}$ Intrauterine contraceptive device are the most commonly used method of reversible contraception worldwide. $^{2}$ Post-placental IUCD has several advantages. The woman is definitely not pregnant and she has high motivation to use contraception at time of immediate postpartum. Among women who have limited access to a clinician, postpartum time provides a unique opportunity to address a woman's need for contraception as the procedure is carried out by experts, and she remains under professional care post-delivery. ${ }^{3}$ It also provides protection against unwanted pregnancy without interfering with breast feeding and avoids discomfort related to insertion. ${ }^{4}$ Family planning 
Division, Ministry of Health and Family Welfare, Government of India promotes provision of IUCD in the immediate postpartum period to address the unmet need of contraception in the Indian population, as stated in the "Postpartum IUCD Reference Manual, November 2010'. Family planning is important not only for population stabilization, but it has been increasingly realized that family planning is central to improve maternal and newborn survival and health. Various studies found that if pregnancies taking place within 24 months of a previous birth have a higher risk of adverse effect like abortion, premature labor, postpartum hemorrhage, low birth weight babies, fetal loss and maternal death. ${ }^{5}$ Due to JSY Scheme Institutional delivery rate has increased and hence more number of patients come to health facility for antenatal and postpartum care and this is the right time to counsel the patient for family planning services. Hence integrating IUCD insertion with delivery services optimizes opportunities for women to obtain an appropriate long-term, reversible family planning method before discharge and during the same hospital stay. In spite of the expulsion rate as high as $8-10 \%$, the retention rate is still $90-92 \%$. (With good technique expulsion rate is reduced to $<3 \%){ }^{6}$

\section{METHODS}

The study was commenced only after obtaining permission from the Institutional Ethics Committee and Maharashtra University of Health and Sciences. Study design was prospective, interventional study, held in department of obstetrics and gynecology at tertiary care center. Counselling about family planning was done in antenatal OPD and indoor ward. Total duration of study was 18 months. Selection of participants done based on following selection criteria.

\section{Inclusion criteria}

- Participants more than or equal to 18 years of age.

- Participants attending the antenatal OPD at tertiary care center

- Participants admitted in the antenatal ward or waiting ward

- Participants fulfilling category 1 and 2 of WHO medical eligibility criteria (MEC) for IUCD insertion ${ }^{7}$

- Participants who are not willing to accept postplacental IUCD insertion.

\section{Exclusion criteria}

- Participants who come under category 3 and 4 of WHO medical eligibility criteria (MEC) for IUCD insertion. $^{7}$

- $\quad$ Premature rupture of membrane $>18$ hours

- Unexplained vaginal bleeding

- Chorioamnionitis
- Distorted uterine cavity (diagnosed by ultrasonography, which will routinely be done antenatally).

\section{Sample size}

Sample size was calculated by following formula:

Sample size $n=\frac{\mathrm{Z} 2(\mathrm{P} \times \mathrm{Q} \times \mathrm{N}) 2}{\mathrm{E} 2(\mathrm{~N}-1)+\mathrm{Z} 2(\mathrm{PQ})}$

$\mathrm{Z}=1.96$ for $95 \%$ confidence level

$\mathrm{P}=$ assuming $50 \%$ acceptance rate

$\mathrm{Q}=100-\mathrm{P}$

$\mathrm{E}=$ Expected allowable error $(5 \%)$

$\mathrm{N}=$ Total no of cases who will be counselled.

(Considering 15 patients counselled per ANC OPD and WWD admission. Hence total number of cases counselled in 18 months $=1440$ ).

Substituting these values, authors get sample size (n) as 282.699 hence 280 .

\section{Study procedure}

- Participants were recruited in the antenatal OPD and antenatal/waiting ward after being evaluated for fulfillment of inclusion and exclusion criteria and informed consent was obtained.

- These participants were counselled for post placental IUCD and various alternative method of contraception like oral contraceptive pills, emergency pill, barrier method, calendar method, injectable progesterone, mini-pills, sterilization etc at the antenatal or waiting ward.

- The advantages and disadvantages of post placental IUCD had been explained to the patient, in detail.

- Total 280 patients were enrolled for study and various variable assessed.

- Acceptability, safety, demographic profile and complications of post-placental insertion of IUCD, were assessed.

- Participants who were accepted post placental IUCD as contraceptive method insertion was done under all aseptic precaution within $10 \mathrm{~min}$ of placental expulsion during vaginal delivery or caesarean section accordingly. Copper-T 380A was used which was available free of cost in the department of obstetrics and gynecology in tertiary care centre as it was supplied by government of India under National family and welfare program.

- During the post-partum period (prior to discharge), the women were given a post placental IUCD information leaflet and explained about the follow-up at 2 weeks, 6 weeks or as soon as she notices any warning signs such as:

- Foul smelling lochia

- Bleeding 
- Any signs and symptoms of infection like fever, myalgia, body ache, discharge per vagina, or pain lower abdomen

- Expulsion of IUCD.

\section{Follow-up}

Follow-up visits were routine as they are postnatal patients, who were routinely called for follow up to 6 weeks.

- Women were asked about any warning symptoms.

- Physical and pelvic examinations were carried out to look for vaginal discharge and forniceal tenderness

- If any of the above was present, the findings were noted and investigator takes care for that and participants were treated medically as appropriate, and were counselled

- Post-placental IUCD thread was checked and trimmed

- In case post-placental IUCD thread was not found on per speculum examination, ultrasound examination was done to confirm the presence of IUCD and managed accordingly.

\section{Statistical analysis}

- Qualitative data was represented in form of frequency and percentage of variables.

- Among qualitative data, nominal data included location (rural/urban), education, occupational status (unemployed/employed), socio-economic status, religion, sex of previous live child, acceptance status (accepted/denied), reason for acceptance and denial, etc.

- In present study association between qualitative variables was assessed by Chi-Square test, with Continuity Correction for all $2 \times 2$ tables and where Chi-Square test was not valid due to small counts analysis done by Fisher's exact test for all $2 \times 2$ tables. In presence of small counts in tables with more than two rows or columns, adjacent row or Column data was pooled and chi-square test reapplied. continuity correction was applied for all $2 \times 2$ tables after pooling of data. Fisher's exact test was applied for all $2 \times 2$ tables where $\mathrm{p}$-value of continuity correction was not valid due to small counts, in-spite of pooling of data (E.g. association between education and acceptance status (accepted/denied).

- In present study quantitative data was represented using mean $\pm \mathrm{SD}$ and median and IQR (interquartile range).

- Comparison of quantitative data measured between binomial qualitative variable (acceptance status (accepted/denied) was done using unpaired t-test, if the data passed 'Shapiro-Wilk test normality test' or by Mann-Whitney U-test if the data failed 'normality' test. (E.g. comparison of age between contraception accepted and denied cases).
- In view of study being cross-sectional study, relative risk was calculated for various risk factors for acceptance status (accepted/denied).

- Results were graphically represented where deemed necessary.

- Appropriate statistical software, including but not restricted to MS excel, PSPP version1.0.1was used for statistical analysis. Graphical representation was done in MS excel package included in microsoft office 365 . An alpha value ( $p$-value) of $<=0.05$ was used as the cut-off for statistical significance.

\section{RESULTS}

In present study 280 cases were enrolled, were distributed on basis of various variables like age, location, education and occupation etc.

Table 1: Distribution among the cases on basis of age (years).

\begin{tabular}{|l|ll|}
\hline Age (years) & No. of patients & Percentage \\
\hline 19 to 23 & 74 & $26.4 \%$ \\
\hline 24 to 28 & 122 & $43.6 \%$ \\
\hline 29 to 33 & 64 & $22.9 \%$ \\
\hline 39 to 43 & 20 & $7.1 \%$ \\
\hline Total & 280 & $100.0 \%$ \\
\hline
\end{tabular}

Age: authors noted maximum cases were age group between 24 to 28 years i.e., 122 cases (43.6\%) (Table $1)$.

Table 2: The distribution among cases on basis of locations.

\begin{tabular}{|lll|}
\hline Location & No. of patients & Percentage \\
\hline Urban & 271 & $96.8 \%$ \\
\hline Rural & 9 & $3.2 \%$ \\
\hline Total & 280 & $100.0 \%$ \\
\hline
\end{tabular}

Location: 271 cases $(96.8 \%)$ were from urban area and only 9 cases $(3.2 \%)$ were from rural area. This difference mainly due to present study conducted in tertiary care, which covers mainly urban areas (Table 2).

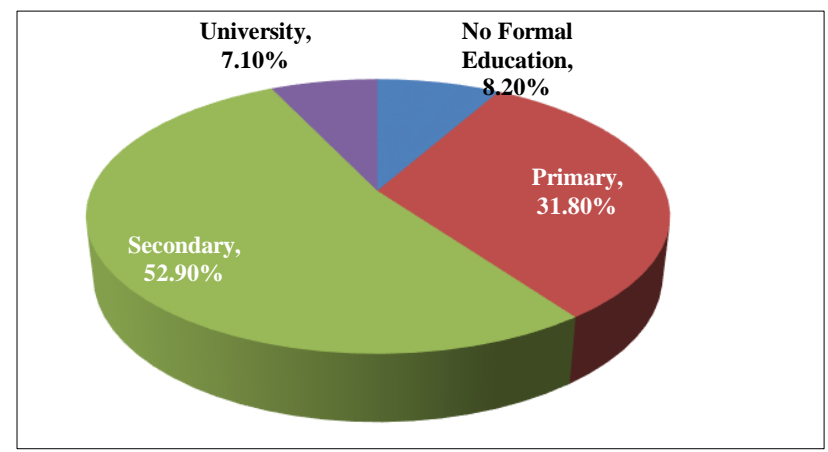

Figure 1: Distribution among the cases on basis of education. 


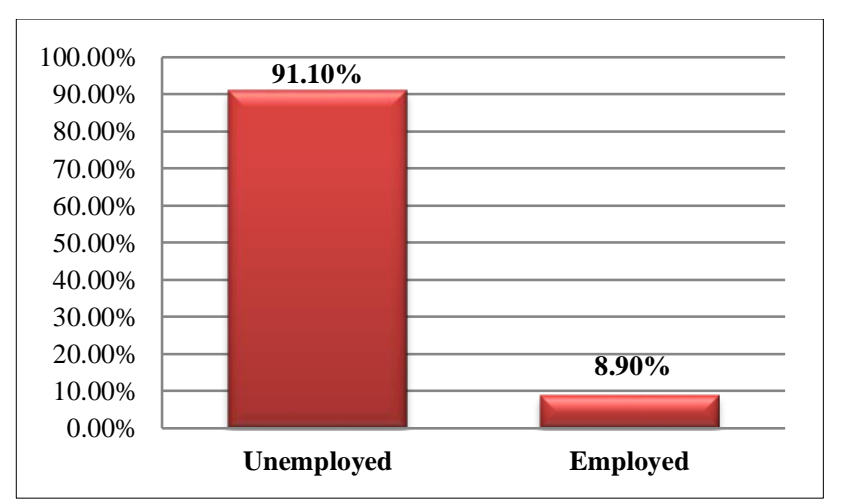

Figure 2: Distribution among the cases on basis of occupation.

Education: authors also noted among all cases maximum cases were educated till secondary education i.e.52.9\%. (Figure 1).

Occupation: 255 cases (91.1\%) unemployed (Figure 2).

Socioeconomic status: On basis of modified Kuppuswammy scale, maximum cases i.e. 201 cases belonged to lower middle-class family (Table 3 ).

Table 3: Distribution among the cases on basis of socioeconomic status.

\begin{tabular}{|lll|}
\hline Socioeconomic status & No. of patient & Percentage \\
\hline Lower & 45 & $16.10 \%$ \\
\hline Upper lower & 11 & $3.92 \%$ \\
\hline Lower middle & 201 & $71.78 \%$ \\
\hline Upper middle & 12 & $4.28 \%$ \\
\hline Upper & 11 & $3.92 \%$ \\
\hline Total & 280 & $100.0 \%$ \\
\hline
\end{tabular}

Religion: among 280 participants, 220 (78.6\%) were Hindu, 59 (21.1\%) were Muslims, 1 (0.4\%) was Christian (Figure 3).

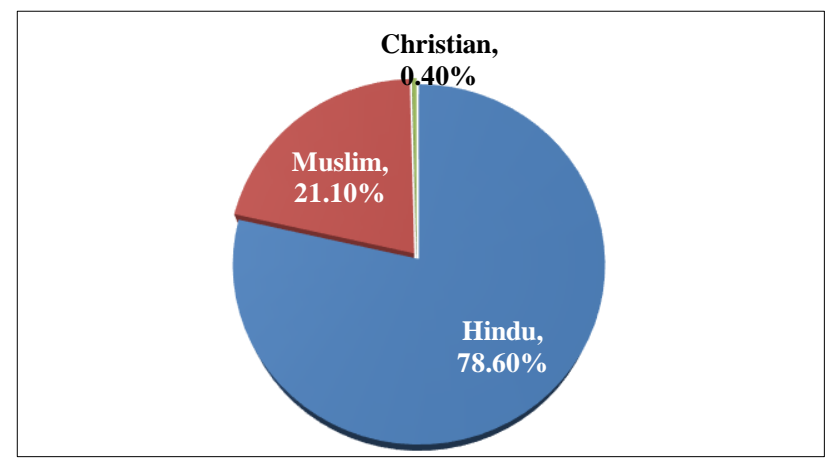

Figure 3: Distribution among the cases on basis of religion.

Parity: among 280 enrolled cases, 159 cases were multigravida i.e. $56.8 \%$ and 121 cases were primigravida i.e. $43.2 \%$ (Figure 4 ).

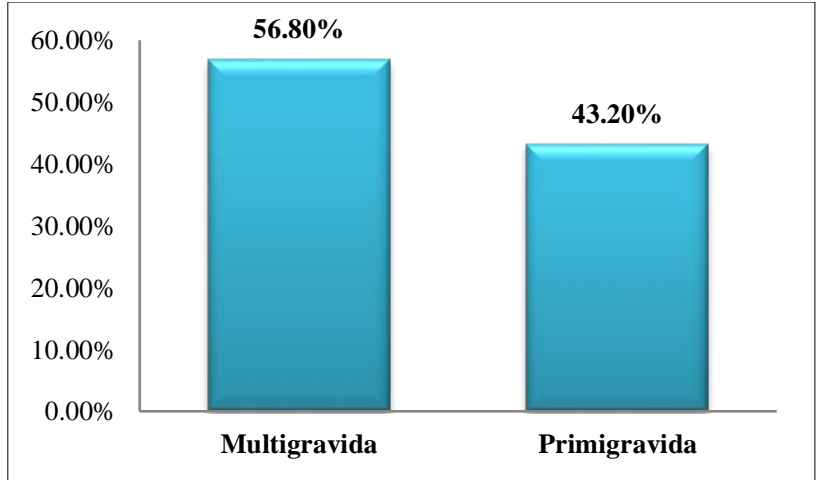

Figure 4: Distribution among the cases on basis of parity.

Table 4: Distribution among the cases on basis of sex of previous live child.

\begin{tabular}{|lll|}
\hline $\begin{array}{l}\text { Sex of previous } \\
\text { live child }\end{array}$ & No. of patients & Percentage \\
\hline Female & 55 & $37.2 \%$ \\
\hline Male & 93 & $62.8 \%$ \\
\hline Total & 148 & $100.0 \%$ \\
\hline
\end{tabular}

Sex of previous live child: among 280 enrolled cases 159 cases were multigravida among them only 148 cases had previous live child. Among 148 cases, 55 cases (37.2\%) had previous female live child and 93 cases $(62.8 \%)$ had previous male child (Table 4).

Table 5: Distribution among the cases on basis of acceptance status.

\begin{tabular}{|lll|}
\hline Acceptance status & No. of patients & Percentage \\
\hline Accepted & 38 & $13.6 \%$ \\
\hline Denied & 242 & $86.4 \%$ \\
\hline Total & 280 & $100.0 \%$ \\
\hline
\end{tabular}

Acceptability: authors noted out of 280 enrolled cases 38 cases $(13.6 \%)$ accepted post placental IUCD as contraception whereas 242 cases $(86.4 \%)$ denied for the same (Table 5).

Table 6: Distribution among the cases on basis of reason for Denial.

\begin{tabular}{|lll|}
\hline Reason for Denial & $\begin{array}{l}\text { No. of } \\
\text { patients }\end{array}$ & Percentage \\
\hline $\begin{array}{l}\text { Don't want contraception } \\
\text { immediately }\end{array}$ & 64 & $26.4 \%$ \\
\hline Fear of complication & 59 & $24.4 \%$ \\
\hline Interested in other method & 58 & $24.0 \%$ \\
\hline Partner has not accepted & 41 & $16.9 \%$ \\
\hline $\begin{array}{l}\text { Complication from } \\
\text { previous use }\end{array}$ & 12 & $5.0 \%$ \\
\hline Religious belief & 8 & $3.3 \%$ \\
\hline Total & 242 & $100.0 \%$ \\
\hline
\end{tabular}


Reason for Denial: authors noted majority (26.4\%) were denied because they do not want contraception immediately, followed by $24.4 \%$ told that they have fear about complication (Table 6).

Table 7: Distribution among the cases on basis of reason for acceptance.

\begin{tabular}{|lll|}
\hline Reason for acceptance & $\begin{array}{l}\text { No. of } \\
\text { patients }\end{array}$ & Percentage \\
\hline Long acting & 14 & $36.8 \%$ \\
\hline Reversible method & 11 & $28.9 \%$ \\
\hline Safe & 6 & $15.8 \%$ \\
\hline Partner not willing to use & 5 & $13.2 \%$ \\
\hline Non-hormonal & 2 & $5.3 \%$ \\
\hline Total & 38 & $100.0 \%$ \\
\hline
\end{tabular}

Reason for acceptance: in present study majority of women (36.8\%) accepted post placental IUCD as contraceptive of choice because of long acting nature of IUCD, followed by $28.9 \%$ women accepted due to reversible method of contraception (Table 7).

Table 8: Distribution among the cases on basis of mode of delivery, who was accepted PPIUCD.

\begin{tabular}{|lll|}
\hline Mode of delivery & No. of patients & Percentage \\
\hline Emergency LSCS & 31 & $81.6 \%$ \\
\hline Elective LSCS & 6 & $15.8 \%$ \\
\hline FTND & 1 & $2.6 \%$ \\
\hline Total & 38 & $100.0 \%$ \\
\hline
\end{tabular}

Mode of delivery: authors noted among 38 women who accepted post placental IUCD, in $31(81.6 \%)$ women insertion done at time of emergency LSCS, in 6 women $(15.8 \%)$ at time of elective LSCS and only in one women insertion done after full term normal vaginal delivery (Table 8).

Table 9: Distribution among the cases on basis of complaints during follow-up visit up to 6 weeks.

\begin{tabular}{|lll|}
\hline $\begin{array}{l}\text { Complaints during } \\
\text { follow-up visit }\end{array}$ & $\begin{array}{l}\text { No. of } \\
\text { patients }\end{array}$ & Percentage \\
\hline Bleeding per vagina & 5 & $13.2 \%$ \\
\hline Pain in abdomen & 2 & $5.3 \%$ \\
\hline Spontaneous expulsion & 1 & $2.6 \%$ \\
\hline No complaint & 30 & $78.9 \%$ \\
\hline Total & 38 & $100.0 \%$ \\
\hline
\end{tabular}

Complication at time of insertion: no complications like uterine perforation, excessive bleeding per vagina etc, occurred at time of insertion of post placental IUCD among all 38 cases.

Complaints during follow-up visit up to 6 weeks: during follow up visit up to 6 weeks post insertion of PPIUCD authors noted among 38 women, 30 women $(78.90 \%)$ had no complaints, 5 women $(13.2 \%)$ had complaints of bleeding per vagina, 2 women $(5.3 \%)$ had complaints of pain in abdomen and one women $(2.6 \%)$ experienced spontaneous expulsion of IUCD within 6 weeks (Table 9).

\section{Association between various variables and acceptance status}

Age: in current study maximum acceptance seen in age group between 24 to $28(16.40 \%)$, however maximum denial $(90.50 \%)$ found age group between 19 to 23 years. (Table 10). In current study association between age of women and acceptance status statistically not significant means age of women not affect the acceptance status.

Table 10: Association among the cases between- acceptance status and age (years).

\begin{tabular}{|lllll|}
\hline \multirow{2}{*}{ Age (years) } & \multicolumn{4}{c}{ Acceptance status } \\
\cline { 2 - 5 } & & Accepted & Denied & Total \\
\hline \multirow{2}{*}{24 to 23} & No. 28 & 7 & 67 & 74 \\
\cline { 2 - 5 } & $\%$ & $9.50 \%$ & $90.50 \%$ & $100.00 \%$ \\
\hline \multirow{2}{*}{29 to 33} & No. & 20 & 102 & 122 \\
\cline { 2 - 5 } & $\%$ & $16.40 \%$ & $83.60 \%$ & $100.00 \%$ \\
\hline \multirow{2}{*}{39 to 43} & No. & 8 & $87.50 \%$ & $100.00 \%$ \\
\hline \multirow{2}{*}{ Total } & $\%$ & $12.50 \%$ & 17 & 20 \\
\hline \multirow{2}{*}{ Chi-square test } & No. & 3 & $85.00 \%$ & $100.00 \%$ \\
\hline Pearson chi-square test & No. & 38 & 242 & 280 \\
\cline { 2 - 5 } & $\%$ & $13.60 \%$ & $86.40 \%$ & $100.00 \%$ \\
\hline
\end{tabular}


Location: in present study association between location and acceptance status was statistically not significant. Relative risk of acceptance of contraception is ratio of probability that urban case accepted, to the probability that urban case denied. Thus, estimate that an acceptance to contraception was 1.007 times more among urban cases than rural cases (Table 11).

Table 11: Association among the cases between- acceptance status and location.

\begin{tabular}{|c|c|c|c|c|}
\hline \multirow{2}{*}{ Acceptance status } & \multicolumn{3}{|c|}{ Location } & \multirow{2}{*}{ Total } \\
\hline & & Urban & Rural & \\
\hline \multirow{2}{*}{ Accepted } & No. & 37 & 1 & 38 \\
\hline & $\%$ & $13.70 \%$ & $11.10 \%$ & $13.60 \%$ \\
\hline \multirow[b]{2}{*}{ Denied } & No. & 234 & 8 & 242 \\
\hline & $\%$ & $86.30 \%$ & $88.90 \%$ & $86.40 \%$ \\
\hline \multirow{2}{*}{ Total } & No. & 271 & 9 & 280 \\
\hline & $\%$ & $100.00 \%$ & $100.00 \%$ & $100.00 \%$ \\
\hline Chi-square test & Value & df & p-value & Association is \\
\hline Pearson chi-square \$ & 0.048 & 1 & 0.827 & Not significant \\
\hline Continuity correction \$ & 0.000 & 1 & 1.000 & Not significant \\
\hline Fisher's exact test & & & 1.000 & Not significant \\
\hline \multirow[t]{2}{*}{ Risk estimate } & Value & \multicolumn{2}{|c|}{$95 \%$ confidence interval } & \\
\hline & & Lower & Upper & \\
\hline For cohort location $=$ urban & 1.007 & 0.951 & 1.066 & \\
\hline For cohort location = rural & 0.796 & 0.102 & 6.187 & \\
\hline
\end{tabular}

Table 12: Association among the cases between- acceptance status and education.

\begin{tabular}{|c|c|c|c|c|}
\hline \multirow{2}{*}{ Education } & & \multicolumn{2}{|c|}{ Acceptance status } & \multirow{2}{*}{ Total } \\
\hline & & Accepted & Denied & \\
\hline \multirow{2}{*}{ No formal education } & No. & 1 & 22 & 23 \\
\hline & $\%$ & $4.30 \%$ & $95.70 \%$ & $100.00 \%$ \\
\hline \multirow{2}{*}{ Primary $^{\wedge}$} & No. & 9 & 80 & 89 \\
\hline & $\%$ & $10.10 \%$ & $89.90 \%$ & $100.00 \%$ \\
\hline \multirow[t]{2}{*}{ Secondary $^{\wedge}$} & No. & 25 & 123 & 148 \\
\hline & $\%$ & $16.90 \%$ & $83.10 \%$ & $100.00 \%$ \\
\hline \multirow{2}{*}{ University^^ } & No. & 3 & 17 & 20 \\
\hline & $\%$ & $15.00 \%$ & $85.00 \%$ & $100.00 \%$ \\
\hline \multirow{2}{*}{ Total } & No. & 38 & 242 & 280 \\
\hline & $\%$ & $13.60 \%$ & $86.40 \%$ & $100.00 \%$ \\
\hline Chi-square test & Value & df & p-value & Association is \\
\hline Pearson chi-square $\$$ & 4.002 & 3 & 0.261 & Not significant \\
\hline Pearson chi-square $^{\wedge}$ & 1.062 & 1 & 0.303 & Not significant \\
\hline 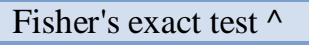 & & & 0.335 & Not significant \\
\hline
\end{tabular}

$\$ 2$ cells (25.0\%) have expected count less than $5 . \wedge$ Row data pooled and chi-square test reapplied with continuity correction, $\wedge 1$ cell $(25.0 \%)$ has expected count less than 5. p-value of Fisher's test will be used.

Education: as per present study there was no significant association between education and acceptance status. However, acceptability more in educated women as compared to non-educated women (Table 12).

Occupation: authors noted there was no significant association between occupation and acceptance status. After estimating relative risk authors estimated that an acceptance to contraception was 1.592 times more among employed cases than unemployed cases (Table 13).

Socioeconomic status: authors noted there was no significant association between socioeconomic status and acceptance status.

However more number acceptance seen in upper lower class (Table 14). 
Table 13: Association among the cases between- acceptance status and occupation.

\begin{tabular}{|c|c|c|c|c|}
\hline \multirow{2}{*}{ Acceptance status } & & \multicolumn{2}{|l|}{ Occupation } & \multirow{2}{*}{ Total } \\
\hline & & Unemployed & Employed & \\
\hline \multirow[t]{2}{*}{ Accepted } & No. & 33 & 5 & 38 \\
\hline & $\%$ & $12.90 \%$ & $20.00 \%$ & $13.60 \%$ \\
\hline \multirow{2}{*}{ Denied } & No. & 222 & 20 & 242 \\
\hline & $\%$ & $87.10 \%$ & $80.00 \%$ & $86.40 \%$ \\
\hline \multirow{2}{*}{ Total } & No. & 255 & 25 & 280 \\
\hline & $\%$ & $100.00 \%$ & $100.00 \%$ & $100.00 \%$ \\
\hline Chi-square test & Value & df & p-value & Association is \\
\hline Pearson chi-square $\$$ & 0.967 & 1 & 0.325 & Not significant \\
\hline Continuity correction $\$$ & 0.459 & 1 & 0.498 & Not significant \\
\hline Fisher's exact test & & & 0.355 & Not significant \\
\hline \multirow{2}{*}{ Risk estimate: acceptance status: accepted/denied } & \multirow{2}{*}{ Value } & \multicolumn{2}{|c|}{ 95\% confidence interval } & \\
\hline & & Lower & Upper & \\
\hline For cohort occupation $=$ unemployed & 0.947 & 0.832 & 1.077 & \\
\hline For cohort occupation $=$ employed & 1.592 & 0.636 & 3.989 & \\
\hline
\end{tabular}

$\$ 1$ cell $(25.0 \%)$ has expected count less than 5. p-value of Fisher's test will be used.

Table 14: Association among the cases between- acceptance status and socioeconomic status.

\begin{tabular}{|llllllll|}
\hline \multicolumn{7}{|c|}{} & \multicolumn{7}{c}{ Socioeconomic status } & Lower & Upper lower & Lower middle & Upper middle & Upper & Total \\
\hline \multirow{2}{*}{ Accepted } & No. & 4 & 3 & 27 & 2 & 2 & 38 \\
\cline { 2 - 8 } & $\%$ & $8.88 \%$ & $27.27 \%$ & $23.43 \%$ & $16.66 \%$ & $18.18 \%$ & $13.60 \%$ \\
\hline \multirow{2}{*}{ Denied } & No. & 41 & 8 & 174 & 10 & 9 & 242 \\
\cline { 2 - 8 } & $\%$ & $91.12 \%$ & $72.73 \%$ & $86.57 \%$ & $83.34 \%$ & $81.82 \%$ & $86.40 \%$ \\
\hline \multirow{2}{*}{ Total } & No. & $4500 \%$ & 11 & 201 & 12 & 11 & 280 \\
\cline { 2 - 8 } & $\%$ & $100.00 \%$ & $100.00 \%$ & $100.00 \%$ & $100.00 \%$ & $100.00 \%$ & $100.00 \%$ \\
\hline Chi-square test & & & Value & Df & p-value & Association is \\
\hline Pearson chi-square $\$$ & & & 2.902 & 4 & 0.574 & Not significant \\
\hline Pearson chi-square & $\wedge$ & & 0.344 & 2 & 0.842 & Not significant \\
\hline
\end{tabular}

$\$ 4$ cells $(40.0 \%)$ have expected count less than $5 .^{\wedge}$ First two and last two column data pooled and chi-square test reapplied.

Table 15: Association among the cases between- acceptance status and religion.

\begin{tabular}{|c|c|c|c|c|c|}
\hline \multirow{2}{*}{ Acceptance status } & & \multicolumn{3}{|l|}{ Religion } & \multirow{2}{*}{ Total } \\
\hline & & Hindu & Muslim ^ & 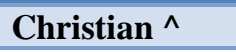 & \\
\hline \multirow{2}{*}{ Accepted } & No. & 26 & 12 & 0 & 38 \\
\hline & $\%$ & $11.80 \%$ & $20.30 \%$ & $0.00 \%$ & $13.60 \%$ \\
\hline \multirow{2}{*}{ Denied } & No. & 194 & 47 & 1 & 242 \\
\hline & $\%$ & $88.20 \%$ & $79.70 \%$ & $100.00 \%$ & $86.40 \%$ \\
\hline \multirow{2}{*}{ Total } & No. & 220 & 59 & 1 & 280 \\
\hline & $\%$ & $100.00 \%$ & $100.00 \%$ & $100.00 \%$ & $100.00 \%$ \\
\hline Chi-square test & Value & df & p-value & Association is & \\
\hline Pearson chi-square \$ & 3.037 & 2 & 0.219 & Not significant & \\
\hline Pearson chi-square $^{\wedge}$ & 2.038 & 1 & 0.153 & Not significant & \\
\hline
\end{tabular}

$\$ 2$ cells $(33.3 \%)$ have expected count less than $5 . \wedge$ Column data pooled and chi-square test reapplied with continuity correction.

Religion: there was no significant association between religion and acceptance status (Table 15). Parity: authors noted there was significant association between parity and acceptance status. Acceptance status was more in multigravida as compared to primigravida. After estimating the relative risk authors noted that an acceptance to contraception was 1.864 times more among multigravida than primigravida (Table 16). 
Table 16: Association among the cases between- acceptance status and parity.

\begin{tabular}{|c|c|c|c|c|}
\hline \multirow{2}{*}{ Acceptance status } & \multicolumn{3}{|c|}{ Parity } & \multirow{2}{*}{ Total } \\
\hline & & Multigravida & Primigravida & \\
\hline \multirow{2}{*}{ Accepted } & No. & 36 & 2 & 38 \\
\hline & $\%$ & $22.60 \%$ & $1.70 \%$ & $13.60 \%$ \\
\hline \multirow{2}{*}{ Denied } & No. & 123 & 119 & 242 \\
\hline & $\%$ & $77.40 \%$ & $98.30 \%$ & $86.40 \%$ \\
\hline \multirow{2}{*}{ Total } & No. & 159 & 121 & 280 \\
\hline & $\%$ & $100.00 \%$ & $100.00 \%$ & $100.00 \%$ \\
\hline Chi-square test & Value & Df & p-value & Association is- \\
\hline Pearson chi-square & 25.805 & 1 & $3.78 \mathrm{E}-07$ & Significant \\
\hline Continuity correction & 24.047 & 1 & $9.40 \mathrm{E}-07$ & Significant \\
\hline \multirow{2}{*}{ Risk estimate: acceptance status: accepted/denied } & \multirow{2}{*}{ Value } & \multicolumn{2}{|c|}{$95 \%$ confidence interval } & \\
\hline & & Lower & Upper & \\
\hline For cohort parity=multigravida & 1.864 & 1.613 & 2.154 & \\
\hline For cohort parity= primigravida & 0.107 & 0.028 & 0.415 & \\
\hline
\end{tabular}

Table 17: Association among the cases between- acceptance status and sex of previous live child.

\begin{tabular}{|lllll|}
\hline \multirow{2}{*}{ Acceptance status } & \multicolumn{4}{l}{ Sex of previous live child } \\
\cline { 2 - 5 } Accepted & \multicolumn{2}{l}{ Female } & Male & Total \\
\hline \multirow{2}{*}{ Denied } & No. & 12 & 24 & 36 \\
\hline \multirow{2}{*}{ Total } & $\%$ & $21.80 \%$ & $25.80 \%$ & $24.30 \%$ \\
\hline Chi-square test & No. & 43 & 69 & 112 \\
\hline Pearson chi-square & $\%$ & $78.20 \%$ & $74.20 \%$ & $75.70 \%$ \\
\hline Continuity correction & No. & 55 & 93 & 148 \\
\hline Risk estimate & $\%$ & $100.00 \%$ & $100.00 \%$ & $100.00 \%$ \\
\hline For cohort sex of previous live child=female & Value & df & p-value & Association is \\
\hline For cohort sex of previous live child=male & 0.299 & 1 & 0.585 & Not significant \\
\hline
\end{tabular}

Table 18: Association among the cases between- birth order and acceptance status.

\begin{tabular}{|c|c|c|c|c|}
\hline \multirow{2}{*}{ Birth order } & & \multicolumn{2}{|c|}{ Acceptance status } & \multirow{2}{*}{ Total } \\
\hline & & Accepted & Denied & \\
\hline \multirow{2}{*}{1} & No. & 2 & 119 & 121 \\
\hline & $\%$ & $1.70 \%$ & $98.30 \%$ & $100.00 \%$ \\
\hline \multirow{2}{*}{2} & No. & 20 & 69 & 89 \\
\hline & $\%$ & $22.50 \%$ & $77.50 \%$ & $100.00 \%$ \\
\hline \multirow{2}{*}{3} & No. & 10 & 43 & 53 \\
\hline & $\%$ & $18.90 \%$ & $81.10 \%$ & $100.00 \%$ \\
\hline \multirow{2}{*}{$4^{\wedge}$} & No. & 5 & 8 & 13 \\
\hline & $\%$ & $38.50 \%$ & $61.50 \%$ & $100.00 \%$ \\
\hline \multirow{2}{*}{$5^{\wedge}$} & No. & 1 & 2 & 3 \\
\hline & $\%$ & $33.30 \%$ & $66.70 \%$ & $100.00 \%$ \\
\hline \multirow{2}{*}{$7^{\wedge}$} & No. & 0 & 1 & 1 \\
\hline & $\%$ & $0.00 \%$ & $100.00 \%$ & $100.00 \%$ \\
\hline \multirow{2}{*}{ Total } & No. & 38 & 242 & 280 \\
\hline & $\%$ & $13.60 \%$ & $86.40 \%$ & $100.00 \%$ \\
\hline Chi-square test & Value & df & p-value & Association is \\
\hline Pearson chi-square $\$$ & 29.954 & 5 & $1.51 \mathrm{E}-05$ & Significant \\
\hline
\end{tabular}




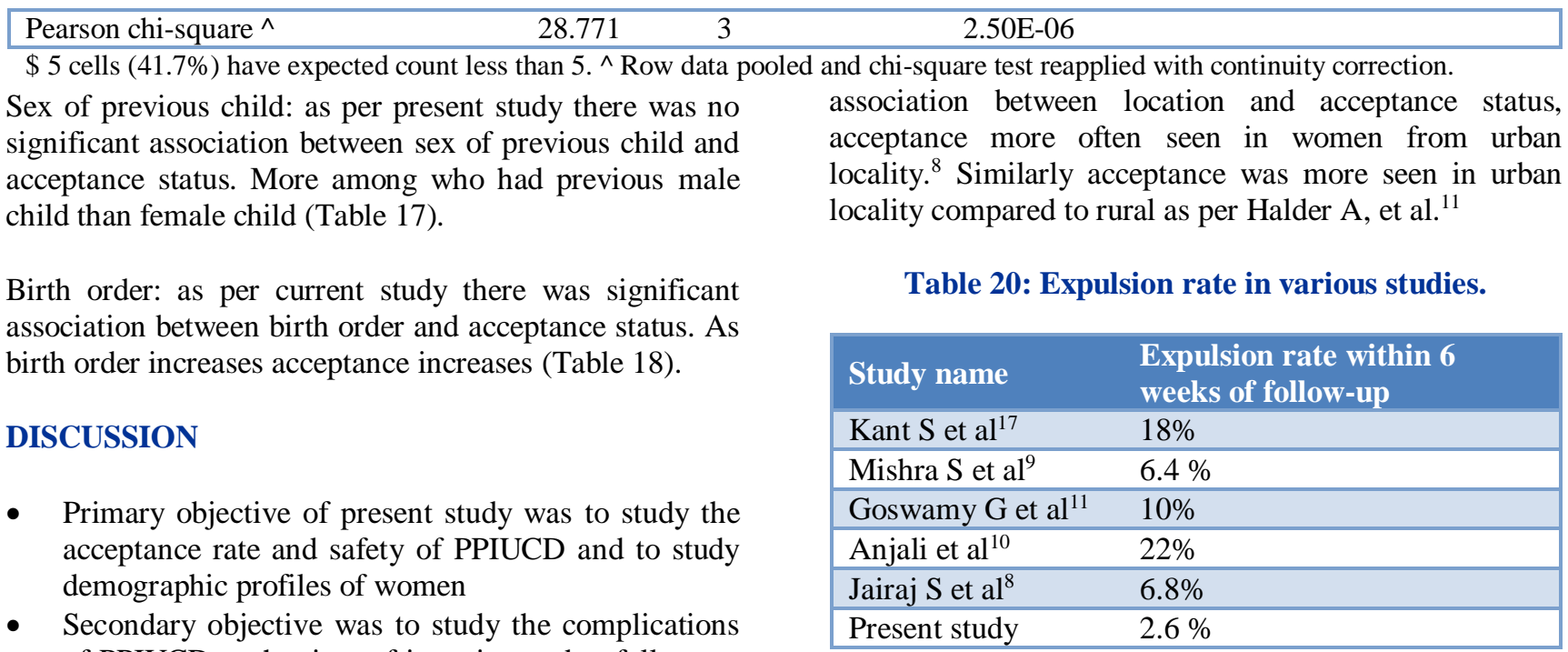
of PPIUCD at the time of insertion and at follow-up visit at 2 weeks and 6weeks and to study the expulsion rate up to 6 weeks of PPIUCD insertion.

\section{Acceptability}

In present study out of 280 women, 38 women (13.57\%) accepted and 242 women $(86.42 \%)$ denied PPIUCD for family planning. Acceptance was so much variable across the country due to difference in locality, sample size and study period as shown in Table 19.

\section{Table 19: Acceptance rate in various studies.}

\begin{tabular}{|c|c|}
\hline Study name & Acceptance rate \\
\hline Jairaj $S$ et al ${ }^{8}$ & $19.72 \%$ \\
\hline Mishra S et al ${ }^{9}$ & $17.57 \%$ \\
\hline Anjali et al $^{10}$ & $36 \%$ \\
\hline Goswamy $\mathrm{G}$ et al ${ }^{11}$ & $66.60 \%$ \\
\hline Vidyaramana et al $^{12}$ & $8.55 \%$ \\
\hline Kant $\mathrm{S}$ et $\mathrm{al}^{13}$ & $39 \%$ \\
\hline
\end{tabular}

Age: in present study mean age of acceptance was $26.82 \pm 3.72$ years. Median age of acceptance was 26 with IQR 5 years. Minimum age of acceptance was 21 years and maximum age 37 years. As per Jairaj et al, mean age of acceptance was $23.70 \pm 2.95$ years. ${ }^{8}$ As per Kumar $S$ et al mean age of acceptance was $24 \pm 4$ years. ${ }^{9}$ As per Valliappan A et al mean age of participants was 25.52 years with a standard deviation (SD) of 4.34 years. ${ }^{10}$ Almost in all study mean age of acceptance is similar.

Although in current study association between age of women and acceptance status was statistically not significant.

Location: in present study association between location and acceptance status was statistically not significant means location not affect the acceptance status. Reason for this type of result mainly due study conducted in area which covered mainly urban population. In contrast, study conducted by Jairaj $\mathrm{S}$ et al, there was significant

Education: as per present study there was no significant association between education and acceptance status. However, acceptability more in educated women as compared to non-educated women. In present study acceptance was more, those completed their secondary education (16.90\%). Whereas non-educated women more prone for denial. Results were similar in study conducted Jairaj $\mathrm{S}$ et al, there was no significant association between education and acceptance status. Although acceptance was more $(23.3 \%)$ in those women who completed their secondary school level education. ${ }^{8}$ As per Mishra S et al, high acceptancy among women who completed their primary and secondary school education. ${ }^{12}$ As per Gunjan Goswamy et al, also found that more acceptance was seen among women who had completed secondary school education (49\%). ${ }^{13}$ Above all studies and current study concluded that educational status has definitely high influence in acceptance.

Occupation: in present study there was no significant association between occupation and acceptance status. After estimating relative risk authors concluded that an acceptance to contraception was 1.592 times more among employed cases than unemployed cases. In contrast, study done by Jairaj $S$ et al, association between occupation and acceptance status was statistically significant and acceptance was high among employed women compared to unemployed women. ${ }^{8}$ Similarly Vidyaramana et al, found employed women high acceptancy $(27.47 \%)$ compared to unemployed. ${ }^{14}$ Above study concluded employed women more aware about family planning so acceptance are high among them.

Socioeconomic status: as per present study there was no significant association between socioeconomic status and acceptance status. However, a greater number of cases, who accepted, belongs to upper lower family. In contrast to current study there was significant association between socioeconomic status and acceptance and acceptance was more in upper middle class as per Jairaj $\mathrm{S}$ et al. ${ }^{8}$ 
Study conducted by Kant S et al, found that low monthly family income was significantly associated with higher acceptance. ${ }^{15}$ Study result may be because study was conducted in government tertiary care hospital where majority women belong to lower class and middle class family.

Parity: as per present study there was significant association between parity and acceptance status. After estimating relative risk authors concluded that an acceptance to contraception was 1.864 times more among multigravida than primigravida. Similar results found in study conducted by Kharkwal $\mathrm{S}$ et al, acceptance was more in multigravida $(57.86 \%)$ compared to primigravida. ${ }^{16}$ Similar result also found in study done by Safwat et al, in Egypt. ${ }^{17}$ Mostly multigravida completed their family, and want family planning. In contrast to above study as per Jairaj $\mathrm{S}$ et al there was no significant association between parity and acceptance, however acceptance was more in primigravida compared to multigravida. ${ }^{8}$ As per Mishra $\mathrm{S}$ et al, high acceptance found among primigravida women $(20.7 \%) .^{12}$

Religion: as per present study no significant association between religion and acceptance status. Result was similar as per study conducted by Jairaj $\mathrm{S}$ et al. ${ }^{8}$

Sex of previous child: as per present study there was no significant association between sex of previous child and acceptance status. Authors estimated that an acceptance to contraception was 1.082 times more among who had previous male child than female child. Kant S.et al found that acceptance rate high in women who had previous male child (40.1\%). ${ }^{15}$ Study conducted by Bhalerao and Purandare stated that the acceptance of PPIUCD was high among women who had at least one male child. ${ }^{18}$

Birth order: as per current study there was significant association between birth order and acceptance status. As birth order increases acceptance increases. In present study highest acceptance $38.50 \%$ seen in 4 th birth order due to beyond that number of participants so much less to construe the result. Similar results found in study conducted by Kant $S$. et al i.e. acceptance rate was gradually increased as the number of previous living children increased. ${ }^{15}$ In contrast to present study Jairaj S. et al found that acceptance was more in who had one child as compared to more than 3 children. ${ }^{8}$

Reason for Denial: authors noted that who denied for PPIUCD insertion, majority were $(26.4 \%)$ did not want contraception immediately, $24.4 \%$ told that they fear about complication, $24 \%$ told they were interested in other method of family planning. As per study conducted by Jairaj $\mathrm{S}$ et al, $63.97 \%$ women denied because they were interested in other method of contraception, $17.17 \%$ women denied because partner not accepted and $10.43 \%$ women denied because they do not want contraception immediately. ${ }^{8}$ As per study conducted by Satyavathi et al, majority were preferred other family planning method
(46.68\%), followed by fear of complications $(32.89 \%)$ and followed by due to family refusal $(20.42 \%) .{ }^{19}$ Reasons for denial in Goswamy $\mathrm{G}$ et al, study were fear of complications $(41 \%)$, non-acceptance by partner $(35 \%) .{ }^{13}$ Anjali et al, found that $32 \%$ want another method of contraception. ${ }^{20}$ Above all studies shows that partner play important role for decision making about contraception hence women's partner should also be counselled during counselling season. As per present study majority women do not want contraception immediately, need further effective counselling regarding family planning, and birth spacing. Women need more motivation about immediate contraception because it reduces maternal and child morbidity.

Reasons for acceptance: authors noted that majority of women (36.8\%) accepted post placental IUCD as contraceptive of choice because of long acting nature of IUCD, followed by $28.9 \%$ women accepted due to reversible method of contraception, $15.8 \%$ women accepted because of its safety profile, $13.2 \%$ women accepted due to partner not willing for other method of contraception. As per Kumar S et al, majority of women $(87.6 \%)$ reported the acceptance of post-placental IUCD as a contraceptive method due it's a long acting nature, $20.5 \%$ considered the non-hormonal nature of the method when choosing. ${ }^{9}$ As per study conducted by Jairaj $\mathrm{S}$ et al, majority of women $(67.12 \%)$ told that they accepted IUCD because a reversible method and $19.17 \%$ accepted because it is long acting temporary contraceptive method. ${ }^{8}$ As per study conducted by Satyavathi et al, reasons for accepting IUCD were long acting (55.28\%), $20.73 \%$ thought it is safe. ${ }^{19}$ As per study conducted by Anjali et al, 28\% accepted because people it is long acting, 20\% accepted because IUCD needs few follow up visits, $17 \%$ because it is reversible. ${ }^{20}$ Above all study supported the fact of post placental IUCD one of the contraceptive method which used as long acting reversible contraceptive method and it is safe. According to report released by WHO in 2006, better family planning and birth-spacing services resulted in better maternal and neonatal outcome. Healthy spacing and timing of pregnancies have a positive effect on maternal health and newborn outcomes. ${ }^{21}$

Mode of delivery in women those accepted post placental IUCD: in present study among 38 women who accepted post placental IUCD, in $31(81.6 \%)$ women insertion done at time of emergency LSCS, in 6 women $(15.8 \%)$ at time of elective LSCS and only in one women insertion done after full term normal vaginal delivery. Similar results found in study conducted by Jairaj S et al, $79.45 \%$ acceptors were who underwent caesarean section and $20.54 \%$ acceptors were delivered vaginally and this difference was statistically significant. $^{8}$ As per study conducted by Shukla $M$ et al, $60.87 \%$ acceptors were who underwent caesarean section. ${ }^{22}$ Vidyaramana et al, found that $83.73 \%$ of acceptors were who had caesarean section and $16.26 \%$ acceptors were who underwent vaginal delivery. ${ }^{14}$ 
Complication at time of insertion: in present study no complication noted during insertion of IUCD. Similar result found in study conducted by Jamkhandi SS et al. No cases of uterine perforation have been reported at time of insertion till now in any literature.

Complaints during follow-up visit up to 6 weeks post insertion: authors noted among 38 women, 30 women (78.90\%) had no complaints, 5 women $(13.2 \%)$ had complaint of bleeding per vagina, 2 women $(5.3 \%)$ had complaint of pain in abdomen and one women $(2.6 \%)$ experienced spontaneous expulsion of IUCD within 6 weeks. Results were similar in study conducted by Jairaj $\mathrm{S}$ et al, majority had $17.17 \%$ bleeding per vagina during follow up within 6 weeks. ${ }^{8}$ Mishra $S$ et al, found that, bleeding $(32.56 \%)$, and pain in abdomen/perineum $(16.28 \%)$ were main complaints during follow-up. ${ }^{12}$ Goswamy $\mathrm{G}$ et al, noted that bleeding/discharge seen in $30 \%$ cases and abdominal pain in $20 \%$ cases. ${ }^{13}$ Anjali et al, observed majority (22\%) were expelled, $8 \%$ had pain abdomen and $6 \%$ found menstrual irregularities. ${ }^{20}$ All above studies and current study observed pain and bleeding per vagina was main complaints during followup which can be managed by proper counselling and treatment.

Comparative to other studies expulsion rate in current study was quite low (Table 20). Upgrading skill by proper training, insertion of IUCD by trained personal, better supervision and proper counselling post insertion can help in reducing the expulsion rate.

\section{CONCLUSION}

PPIUCD is one of the best long acting reversible contraceptive methods. It does not affect breast milk production. Woman does not need extra visit to clinic for contraception and she is ensured that she has adequate contraception before getting discharge from hospital. PPIUCD provide adequate birth spacing between two children which reduces maternal and child morbidity. In present study there was statistically significant association between parity, birth order and age of youngest child. However, literacy, urban locality and employment had positive influence over acceptance. If good technique of insertion will be used than expulsion rate will also reduce as seen in present study i.e. expulsion rate only $2.6 \%$.

Funding: No funding sources

Conflict of interest: None declared

Ethical approval: The study was approved by the Institutional Ethics Committee

\section{REFERENCES}

1. Hall JE. The female reproductive system, infertility and contraception, Harrison's principles of internal medicine, $18^{\text {th }}$ edition: chapter; 2012;347:3037-38.
2. Contraception C. In: Cunningham F. Gray, Leveno KJ, Bloom SL, eds. Willams obstetrics. $25^{\text {th }}$ ed. New York: McGraw-Hill; 2018:680-702.

3. Kapp N, Curtis KM. Intrauterine device insertion during the postpartum period: a systematic review. Contracept. 2009;80:327-36.

4. Grimes DA, Lopez LM, Schulz KF, Van Vliet HAAM, Stanwood NL. Immediate post-partum insertion of intrauterine devices. Cochrane Database Syst Rev. 2003;(1):CD003036.

5. Kanhere A, Pateriya P, Jain M. Acceptability and feasibility of immediate post-partum IUCD insertion in a tertiary care centre in Central India. Int J Reprod Contracept Obstet Gynecol. 2015;4(1):1.

6. Family Planning Division, Ministry of Health and Family Welfare, Government of India; NewDelhi. Fact sheet: Postpartum IUCD, Reference Manual. November 2010. Available at: http://www.nrhmtn.gov.in/modules/PPIUCD\%20Ref erence\%20Manual.pdf. Accessed on $12^{\text {th }}$ March 2020.

7. World health organization, Geneva. Fact sheetmedical eligibility criteria for contraceptive use, $5^{\text {th }}$ ed; $2015 . \quad$ Available at: https://www.who.int/reproductivehealth/publications /family_planning/Ex-Summ. Accessed on $24^{\text {th }}$ March 2020.

8. Jairaj S, Dayyala S. A cross sectional study on acceptability and safety of IUCD among postpartum mothers at tertiary care hospital, Telangana. J Clin Diagn Res. 2016;10(1):LC01-4.

9. Kumar S, Sethi R, Balasubramaniam S. Women's experience with postpartum intrauterine contraceptive device use in India. Reprod Health. 2014;11:32.

10. Valliappan A, Dorairajan G, Chinnakali P. Postpartum intrauterine contraceptive device: Knowledge and factors affecting acceptance among pregnant/parturient women attending a large tertiary health center in Puducherry, India. Int J Adv Med Health Res. 2017;4:69-74.

11. Halder A, Sowmya MS, Gayen A, Bhattacharya P, Mukherjee S, Datta S. A prospective study to evaluate vaginal insertion and intra-cesarean insertion of post-partum intrauterine contraceptive device. J Obstet Gynaecol India. 2014;66(1):35-41.

12. Mishra S. Evaluation of safety, efficacy, and expulsion of post-placental and intra-cesarean insertion of intrauterine contraceptive devices (PPIUCD). J Obstet Gynaecol India. 2014;64(5):33743.

13. Goswami G, Yadav K, Patel A. A prospective study to evaluate safety, efficacy and expulsion rate of post placental insertion of intra uterine device. Journal of Evolut Med Dent Sci. 2015;4(56):9770-5.

14. Vidyarama R, Nagamani T, Prasad K. PPIUCD as a long acting reversible contraceptive (Larc): an experience at a tertiary care centre. Int $\mathbf{J}$ of Scient Res. 2015;4:5-7. 
15. Kant S, Archana S, Singh AK, Ahamed F, Haldar P. Acceptance rate, probability of follow-up, and expulsion of postpartum intrauterine contraceptive device offered at two primary health centers, North India. J Family Med Prim Care. 2016;5(4):770-6.

16. Kharkwal S, Sharma S, Kumari M. A comparative study of postpartum intrauterine contraceptive device acceptance between primiparous and multiparous women in a tertiary care centre. Int $\mathbf{J}$ Reprod Contracept Obstet Gynecol. 2016;5:2304-6.

17. Mohamed SA, Kamel MA, Shaaban OM, Salem HT. Acceptability for the use of postpartum intrauterine contraceptive devices: the assiut experience. Med Princ Pract. 2003;12(3):170-5.

18. Bhalerao AR, Purandare MC. Post-puerperal $\mathrm{Cu}-\mathrm{T}$ insertion: a prospective study. J Postgrad Med. 1989;35:70-3.

19. Satayatietal Maluchuru S, Aruna V. Post-partum intrauterine device insertion - 2 year experience at a tertiary care center in Guntur Medical College /Govt. General Hospital, Guntur. J Dent Med Sci Ver IV. 2015;14(7):2279-861.
20. Kanhere A, Pateriya P, Jain M. Acceptability and feasibility of immediate post-partum IUCD insertion in a tertiary care centre in Central India. Int J Reprod Contracept Obstet Gynecol. 2015;4(1):1.

21. Maternal and Child Health Integrated Program and PPFP activities, WHO Report. Available at: http://www.k4health.org/sites/default/ files/PPFPMeetingReport. Accessed on $12^{\text {th }}$ March 2020.

22. Shukla M, Qureshi S, Chandrawati. Post-placental intrauterine device insertion - a five-year experience at a tertiary care centre in North India. Indian J Med Res. 2012;136:432.

Cite this article as: Jain N, Choudhari HK. A prospective study of acceptability, safety and demographic profile for post placental intrauterine contraceptive device. Int J Reprod Contracept Obstet Gynecol 2020;9:2674-85. 